\title{
Estructura del estrato arbóreo circundante de un cenote, km 37 carretera Mahahual-Xcalak, Quintana Roo, México
}

\author{
Alicia del Socorro Coronado Ávila* \\ José Manuel Castro Pérez \\ Ricardo Torres Lara
}

\section{Resumen}

Se analizó la estructura y diversidad de especies arbóreas en cuatro unidades de muestreo (им) de 50 x 50 m ( 0.25 ha), cada una establecida al azar en cada punto cardinal con referencia al cenote. Se registraron variables dasométricas de todos los individuos con diámetro a la altura del pecho $\geq 5 \mathrm{~cm}$, determinando su estratificación vertical y distribución horizontal, así como el cálculo del índice de importancia estructural y de diversidad. La vegetación presentó tres estratos arbóreos bien definidos: inferior $(<4 \mathrm{~m})$, medio $(4.1-6 \mathrm{~m})$ y superior $(>6 \mathrm{~m})$, mientras que la distribución horizontal de los individuos fue generalmente agregada. Se identificaron 16 especies pertenecientes a 12 familias; la Combretaceae fue la más rica en especies (3), seguida de Polygonaceae (2) y Sapotaceae (2). Las especies Bucida buceras (Linneo), Conocarpus erectus (Linneo) y Chrysobalanus icaco var. Pellocarpus (Linneo) tuvieron los valores más altos de importancia estructural. De acuerdo con el índice de Shannon-Wiener (H') fue mayor en la um2 que en las uм1, uм3 у им4. Se utilizó un análisis de agrupamiento para clasificar la vegetación, y fueron las características del hábitat las que contribuyeron con las especies distintivas de cada área.

Palabras clave

Vegetación, estructura de la comunidad, diversidad, clasificación de la vegetación.

*Correo electrónico: alicia_coronado1989@hotmail.com 


\title{
The arboreal layer structure surrounding a cenote, km. 37 Mahahual-Xcalac highway, Quintana Roo, Mexico
}

\author{
Alicia del Socorro Coronado Ávila* \\ José Manuel Castro Pérez \\ Ricardo Torres Lara
}

\begin{abstract}
The arboreal structure and species diversity in four 50 x $50 \mathrm{~m}$ (0.25 ha) sampling locations (UM) were analyzed, each randomly established at each cardinal point relative to the cenote. Dasonomic variables of every individual with a diameter at breast height of $\geq 5 \mathrm{~cm}$ were registered, determining their vertical stratification and horizontal distribution as well as the estimation of the structural importance and diversity indexes. The vegetation presented three well-defined arboreal strata: lower $(<4 \mathrm{~m})$, medium (4.1-6 m) y superior $(>6 \mathrm{~m}$ ) while the horizontal distribution of individuals was in general aggregated. 16 species belonging to 12 families were identified; Combretaceae was the richest in species (3) followed by Polygonaceae (2) y Sapotaceae (2). Bucida buceras (Linneo), Conocarpus erectus (Linneo) and Chrysobalanus icaco var. Pellocarpus (Linneo) species had the highest structural importance values. According to the Shannon-Wiener index, (H') was highest in UM2 than in UM1, UM3 y UM4. A cluster analysis was used to classify the vegetation and the natural habitat characteristics contributed to the distinctive species en each area.
\end{abstract}

KEY WORDS

Vegetation, community structure, diversity, vegetation classification.

*E-mail: alicia_coronado1989@hotmail.com 


\section{Introducción}

Los bosques y selvas proveen gran variedad de beneficios directos e indirectos, entre los que se encuentra la regulación del ciclo hidrológico, pues equilibran la precipitación en una zona por medio de la transpiración; igualmente mantienen con sus raíces y hojarasca condiciones apropiadas para una infiltración gradual de la lluvia en el suelo, la cual favorece la recarga de los acuíferos y el mantenimiento de los ríos y manantiales, impidiendo la formación de escurrimientos superficiales que en grandes volúmenes ocasionan la erosión del suelo (Karousakis, 2007). Uno de los problemas básicos en ecología es establecer las causas que determinan la distribución y abundancia de los organismos (Andrewartha y Birch, 1954). La amplitud en su distribución depende de una gama de factores tanto físicos como químicos (Krebs, 1978); la tolerancia de las especies ante ellos es trascendental, ya que el establecimiento, desarrollo y reproducción de los individuos puede estar limitada por los mismos (Andrewartha y Birch, 1954; Krebs, 1978; Moreno-Casasola, 1986). El efecto de estos factores puede estudiarse con relativa sencillez en sistemas con baja riqueza de especies vegetales y no debe sorprender que la vegetación pueda ser más fácilmente interpretada como un mosaico asociado con ciertas características del ambiente (Jiménez-Bueno, 1994). Según Campos-Cámara (1996) Quintana Roo, casi en su totalidad, es cubierta por selva, y una mínima parte (10\%) corresponde a manglar y tular. La vegetación distribuida en el estado es selva mediana subperennifolia, mientras que la franja costera de la entidad se distingue por presentar condiciones de inundación o sequía pronunciada durante la estación más seca del año, lo cual trae como resultado el establecimiento de vegetación compuesta por flora tolerante a la salinidad, caracterizada por su baja diversidad. La franja costera es de suma relevancia turística para el sur del estado, ya que representa una fuente de empleos con una importante derrama económica, lo que ha propiciado la explotación de recursos naturales durante muchos años, y ha generado en la última década que el área haya exhibido un rápido crecimiento demográfico con la consecuente urbanización de zonas naturales. Esa zona posee humedales, cenotes, lagunas costeras, esteros, aguadas, sartenejas y lagunas arrecifales, que albergan gran diversidad biológica. El área de estudio presenta vegetación de trascendencia ecológica como los manglares, los cuales funcionan en calidad de hábitat de un elevado número de especies, además de que juegan un papel cardinal en el funcionamiento de 
los sistemas costeros tropicales a través de la exportación de materia orgánica. Asimismo está destinada a la urbanización, lo que le da primordial importancia a este trabajo, ya que al haber un cenote que tiene contacto con el área de la sabana este sitio es relevante para la conservación de especies de flora y fauna que ahí habitan. Por lo tanto, el objetivo de la investigación fue definir la estructura de la comunidad del estrato arbóreo en cuatro unidades de muestreo (UM) circundantes a un cenote mediante la determinación de la distribución espacial, la jerarquización de la dominancia de especies y la caracterización de la heterogeneidad del estrato arbóreo en las um. Los resultados del presente trabajo servirán para establecer las condiciones en las que se encuentra la vegetación arbórea de acuerdo con los cuatro factores que se hallaron en los puntos de muestreo (al lado norte, una construcción; al lado sur, un rancho; al lado oeste, la sabana, y al lado este, la carretera); esto constituirá un antecedente para futuros estudios, en los cuales se involucren otros factores para determinar la factibilidad de un reordenamiento en cuanto a la urbanización que se está dando en la zona.

\section{Antecedentes}

Los cenotes poseen una gran diversidad ecológica, en la cual la vegetación circundante depende de la topografía del terreno, el tipo de suelo y el clima del lugar (Medina-González, 2000). Tal vegetación está conformada por especies de las zonas externas, pero que presentan mayor perennifoliedad y poseen característicamente muchas epífitas. En la actualidad no existen trabajos sobre vegetación asociada con cenotes realizados en la zona de interés, pero se pueden mencionar estudios como el de Zaldívar-Jiménez (1999), quien definió la vegetación de manglar en la ciénaga de Sisal-Chuburná y su relación con características físicas y químicas del sedimento, determinando que la salinidad repercute sobre la estructura, el desarrollo del manglar y la vegetación circundante. Cortés-Castelán e Islebe (2005) abordaron la influencia de factores ambientales vinculados con la distribución de especies arbóreas en selvas del sureste de México, resultando la presencia de especies con distribución restringida a zonas bajas inundables como Crescentia cujete y Ouratea nítida. Tun-Dzul et al. (2008) investigaron la estructura arbórea en los bajos inundables de la Península de Yucatán, donde registraron un total de 7176 individuos, una diversidad de 3.02 bits/individuo y diferencias en la composición de la vegetación de acuerdo 
con índices de semejanza florística. Otro trabajo importante y relacionado con el presente estudio es el realizado por Zarco-Espinosa et al. (2010), en el cual se analizó la estructura y diversidad de especies arbóreas en el Parque Estatal Agua Blanca, Macuspana, Tabasco, y se obtuvieron 71 especies con dos estratos bien definidos, una distribución generalmente aleatoria y una diversidad intermedia en comparación con otras selvas de México.

\section{Materiales y métodos}

\section{Área de estudio}

El área de estudio se ubica en el km 37 de la carretera Mahahual-Xcalak, localizada al sur del estado de Quintana Roo, en el municipio de Othón P. Blanco, entre las coordenadas $18^{\circ} 26^{\prime} 41.44^{\prime \prime}$ latitud norte y $87^{\circ} 47^{\prime} 56.58^{\prime \prime}$ longitud oeste (Arriaga-Cabrera et al., 1998) (figura 1). El área tiene un clima tropical subhúmedo con lluvias en verano y parte del invierno; la precipitación promedio es de 1400 $\mathrm{mm}$ al año. En función de lo anterior, suelen reconocerse tres épocas climáticas: secas, lluvias y nortes. La región exhibe diferentes tipos de vegetación, entre los que destacan la selva baja inundable, integrada por una comunidad arbórea que presenta el dosel semicerrado, y las asociaciones de manglares, siendo estos los de mayor cobertura debido a la naturaleza inundable de los suelos que dominan en la región. Los ejemplares más comunes de manglar son Rhizophora mangle, Conocarpus erectus y Avicennia germinans, cuyas alturas varían entre 5 y $10 \mathrm{~m}$ (Cabrera, 1997: 24).

\section{Unidades de muestreo}

En el área de estudio se establecieron cuatro um de 50 x 50 m (0.25 ha) cada una, tomadas de acuerdo con los puntos cardinales (norte, sur, este y oeste). Cada um se dividió en 25 cuadros de 10 x $10 \mathrm{~m}\left(100 \mathrm{~m}^{2}\right)$. En cada cuadro se localizaron mediante coordenadas cartesianas $(\mathrm{x}, \mathrm{y})$ todos los individuos de las especies arbóreas con diámetro a la altura del pecho (DAP) $\geq$ a $5 \mathrm{~cm}$ tomado con cinta diamétrica, y se posicionaron con un GPs según lo sugerido por Hubbell (1979), Hubbell y Foster (1983) y Dallmeier (1992) para bosques tropicales. A cada individuo se le asignó un número y fueron marcados con pintura en aerosol color naranja. En cada um se midió diámetro del tallo (a $1.30 \mathrm{~m}$ de altura a partir de la base-DAP- de 
los individuos $\geq 5 \mathrm{~cm}$ de diámetro), diámetro de la copa (DC), altura de fuste (AF) y altura de las especies arbóreas con un distanciómetro de mano marca Leica, y se registraron los nombres común y científico (Mueller-Dombois y Ellenberg, 1974; Cox, 1981). Respecto aquellos individuos que no se reconocieron en campo, fueron procesados (Lot y Chiang, 1986) e identificados en el herbario del Centro de Investigación Científica de Yucatán (CICY) de Mérida, con la colaboración del doctor Germán Carnevali Fernández Concha.

Asimismo, para analizar la altura, esta se agrupó en tres estratos arbóreos de acuerdo con la altura promedio: a) superior, con individuos $>6 \mathrm{~m}$ de altura; b) medio, entre 4.1 y $6 \mathrm{~m}$ de altura, y c) inferior, $<4 \mathrm{~m}$ de altura. Con los datos obtenidos se calculó diámetro relativo, altura relativa y cobertura relativa, con los cuales se determinó el índice de valor forestal (IvF) (Curtis y Mclntosh, 1951) para cada um.

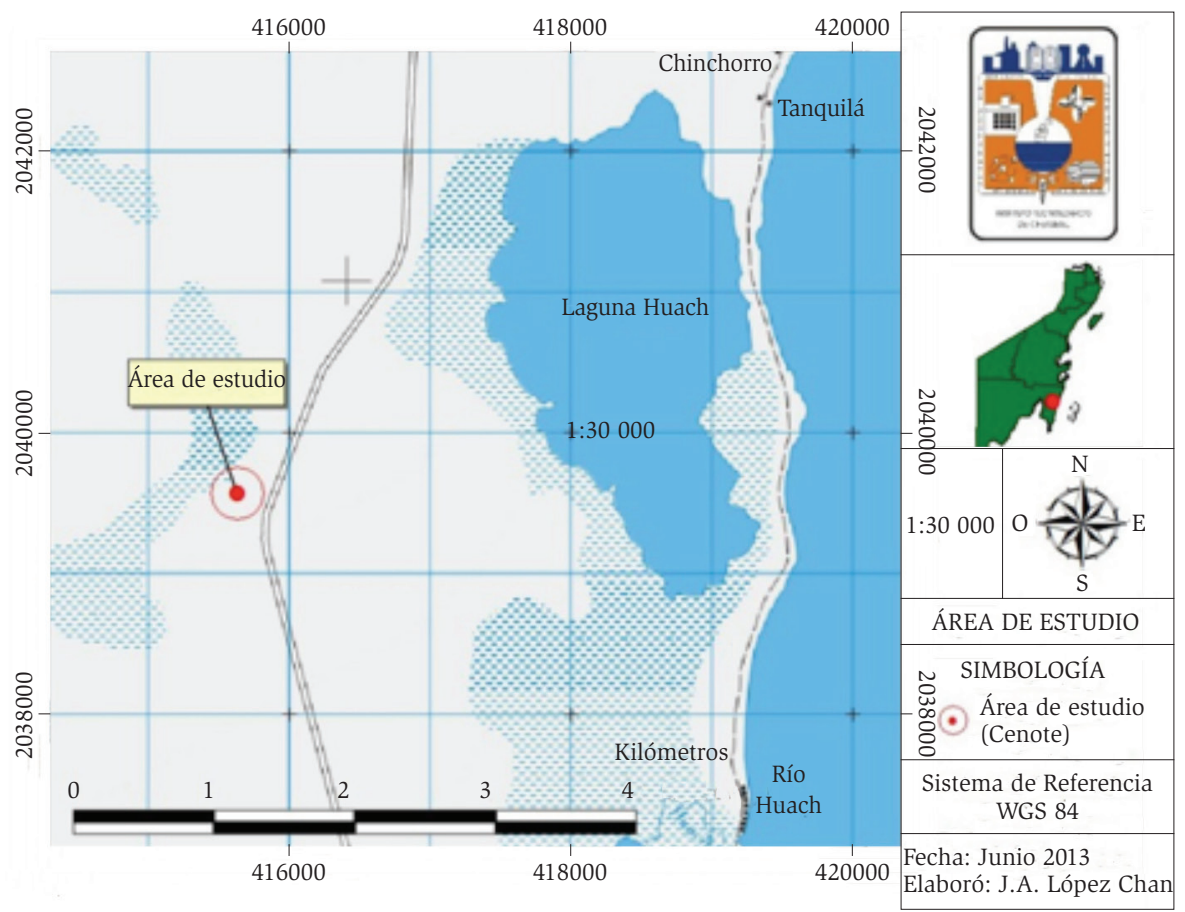

Figura 1. Mapa del área de estudio (programa ArcGIS 9.2) 
El IvF se aplicó con el propósito de evaluar la estructura bidimensional del estrato arbóreo en la zona de estudio considerando tres medidas: la primera al nivel del estrato inferior en el plano horizontal (DAP), la segunda incluye los estratos inferior y superior en el plano vertical (altura), y la tercera al nivel del estrato superior en el plano horizontal (cobertura). El índice se calculó como sigue:

$$
I V F=\text { Diámetro relativo }+ \text { Altura relativa }+ \text { Cobertura relativa }
$$

Donde el diámetro, la altura y la cobertura relativos se obtuvieron de la siguiente manera:

$$
\begin{aligned}
\text { Diámetro relativo } & =\frac{\text { Diámetro absoluto de cada especie }}{\text { Diámetro absoluto de todas las especies }} \times 100 \\
\text { Altura relativa } & =\frac{\text { Altura absoluta de cada especie }}{\text { Altura absoluta de todas las especies }} \times 100 \\
\text { Cobertura relativa } & =\frac{\text { Cobertura absoluta de cada especie }}{\text { Cobertura absoluta de todas las especies }} \times 100
\end{aligned}
$$

Estos índices fueron definidos para cada UM (0.25 ha) de manera independiente. Por otra parte, a cada una se le determinó su riqueza específica y la contribución de la abundancia relativa de sus especies a través del cálculo de la diversidad de Shannon-Wiener logaritmo base 2 y la equidad de Pielou (Clarke y Warwick, 2001). Por último, para detectar patrones similares entre las UM se empleó el coeficiente de similitud de Bray-Curtis, y posteriormente se realizó un análisis del porcentaje de similitud (SIMPER) (Clarke y Warwick, 2001) para identificar a las especies que se discriminan entre las UM. El procedimiento SIMPER examinó la contribución de cada especie al promedio de similitud de Bray-Curtis. Todas las técnicas multivariadas fueron efectuadas con el programa computacional PRIMER 5 (Plymouth Laboratory) (Clarke y Gorley, 2006). 


\section{Resultados}

En el cuadro 1 se muestra la lista florística correspondiente a la vegetación circundante del cenote. En total, se registraron 16 especies del estrato arbóreo, pertenecientes a 12 familias. Dentro de estas últimas destacan Combretaceae (3 especies), Poligonaceae (2) y Sapotaceae (2). Es importante subrayar que las principales especies arbóreas, como Bucida buceras (Linneo), Conocarpus erectus (Linneo), Metopium brownei (Jacq.) Urban y Chrysobalanus icaco var. pellocarpus (Linneo) se distribuyen ampliamente en esta selva. Sin embargo, otras como Rhizophora mangle (Linneo), Manilkara zapota L. (P. Royen), Coccoloba cozumelensis (Hemsl) y Coccoloba acuminata (Kunth) se presentan de manera discontinua en rodales con variadas condiciones ambientales o con diferente antigüedad.

Cuadro 1. Composición florística de las cuatro unidades de muestreo en el área de estudio

\begin{tabular}{|c|c|c|}
\hline FAMILIA & ESPECIES & NOMBRE COMÚN \\
\hline Annonacea & Annona glabra (Linneo, 1953) & Corcho \\
\hline Combretaceae & $\begin{array}{l}\text { Bucida buceras (Linneo, 1959) } \\
\text { Conocarpus erectus (Linneo, 1953) } \\
\text { Laguncularia racemosa (Linneo, 1959; Gaertn. } \\
\text { f.) }\end{array}$ & $\begin{array}{l}\text { Pucté } \\
\text { Mangle } \\
\text { botoncillo } \\
\text { Mangle blanco }\end{array}$ \\
\hline Chrysobalanaceae & $\begin{array}{l}\text { Chrysobalanus icaco var. pellocarpus (Linneo, } \\
\text { 1817) }\end{array}$ & Icaco \\
\hline Polygonaceae & $\begin{array}{l}\text { Coccoloba acuminata (Kunth, 1817) } \\
\text { Coccoloba cozumelensis (Hemsl, 1887) }\end{array}$ & $\begin{array}{l}\text { Sak-boob } \\
\text { Boob }\end{array}$ \\
\hline Bignoniaceae & Crescentia cujete (Linneo, 1953) & Jícara \\
\hline Erythroxylaceae & Erytroxylum areolatum (Linneo, 1959) & Indio \\
\hline Theophrastaceae & Jacquinia aurantiaca (W. T. Aiton, 1811) & Limoncillo \\
\hline Sapotaceae & $\begin{array}{l}\text { Manilkara zapota (Linneo, 1953; P. Royen) } \\
\text { Sideroxylon amygdalinum (Standl, 1932) }\end{array}$ & $\begin{array}{l}\text { Zapote } \\
\text { Silion }\end{array}$ \\
\hline Anarcadiaceae & Metopium brownie (Jacq. Urb, 1908) & Chechén \\
\hline Myricaceae & Myrica cerifera (Linneo, 1953) & Árbol de cera \\
\hline Ochnaceae & Ouratea nítida (Sw. Engl ,1876) & Cabanite \\
\hline Rhizophoraceae & Rhizophora mangle (Linneo, 1953) & Mangle rojo \\
\hline
\end{tabular}

Fuente: Elaboración propia. 


\section{Estructura horizontal}

La mayoría de los individuos de las especies presentaron diámetros $<10 \mathrm{~cm}$ concentrados en la clase diamétrica de $5-10 \mathrm{~cm}$, con un total de 690 individuos pertenecientes a las cuatro um (figura 2). Las especies reportadas para esta categoría fueron Conocarpus erectus, Chrysobalanus icaco var. pellocarpus, Myrica cerifera (Linneo), Rhizophora mangle y Bucida buceras. En la clase diamétrica de 10.1-15 cm se ubicaron 308 individuos, cuyas especies más representativas fueron Conocarpus erectus, Chrysobalanus icaco var. pellocarpus, Bucida buceras, Metopium brownei y Myrica cerifera. En las categorías mayores solo se hallaron cinco individuos, pertenecientes principalmente a Bucida buceras y Chrysobalanus icaco var. pellocarpus.

\section{Estructura vertical}

ELa mayor altura alcanzada del estrato arbóreo fue de $8 \mathrm{~m}$, diferenciándose tres estratos: inferior, medio y superior (figura 2). En el estrato inferior, constituido por alturas menores a $4 \mathrm{~m}$, se registró el mayor número de individuos (678), representado por las especies $C$. icaco var. pellocarpus, $C$. erectus, $M$. cerifera, $R$. mangle y $M$. brownei, y el estrato con mayor altura $>6 \mathrm{~m}$ exhibió el menor número de individuos (109), pertenecientes generalmente a Bucida buceras y $C$. icaco var. pellocarpus. Por um, la 1 dominó el estrato inferior (294 individuos), la 3 el estrato medio (188) y la 4 el estrato superior (38).

Índice estructural

Las especies con altos índices de valor forestal presentes en las cuatro um fueron $B$. buceras, $C$. erectus y C. icaco var. pellocarpus (cuadro 2). Las um 2, 3 y 4 compartieron Bucida buceras con alto IVF, mientras que C. erectus se encontró en las um 1, 2 y 3. Por um, las especies con mayor valor forestal fueron Chrysobalanus icaco var. pellocarpus y Conocarpus erectus para la 1, Bucida buceras y Conocarpus erectus para la 2 y la 3 , y en la 4 las especies que más contribuyeron con el porcentaje acumulado fueron Bucida buceras y Chrysobalanus icaco var. pellocarpus. 


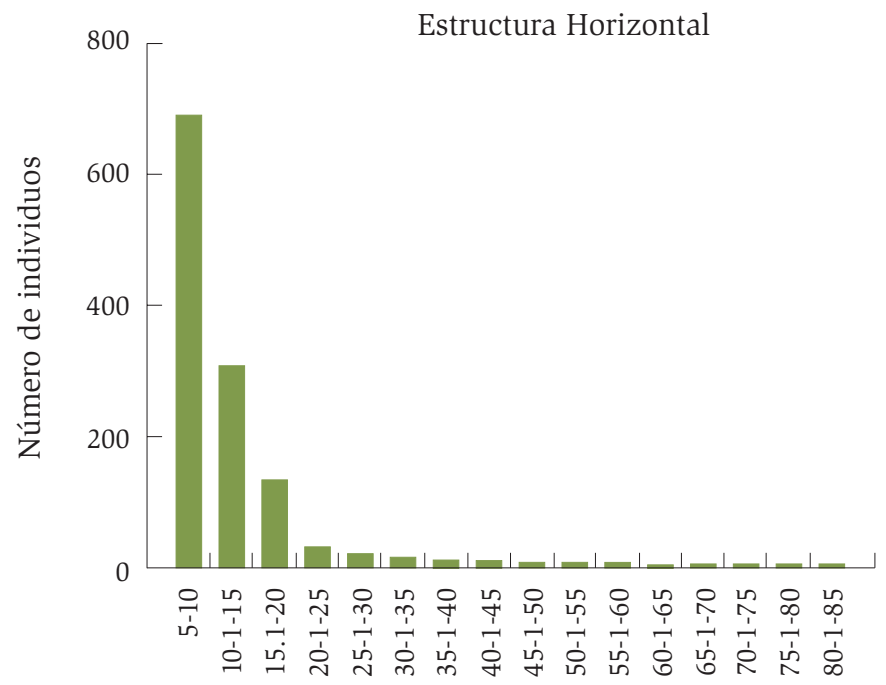

Diámetro a la altura del pecho $(\mathrm{cm})$

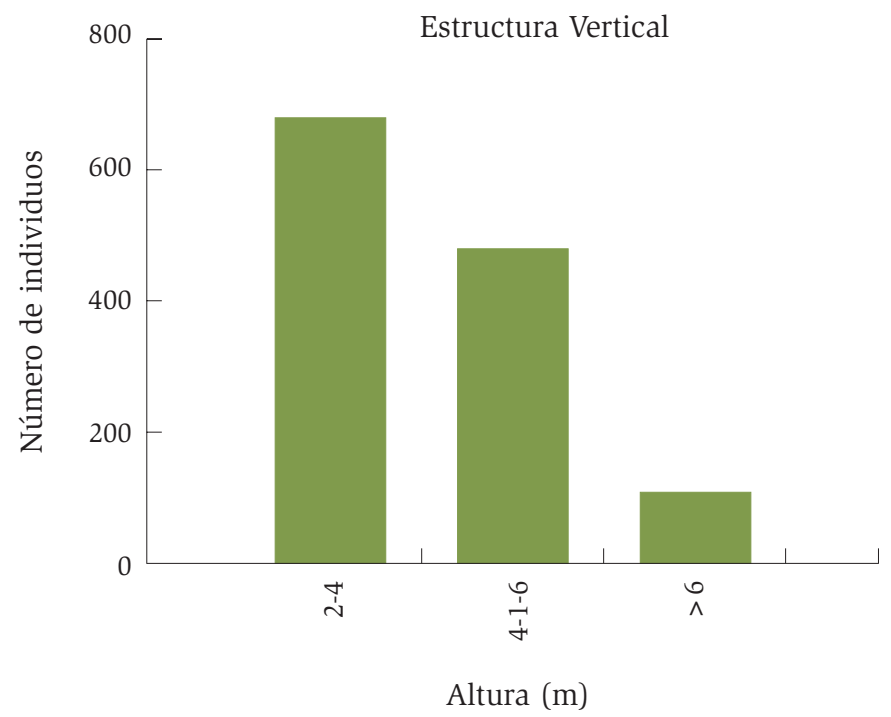

Fuente: Elaboración propia.

Figura 2. Estratificación horizontal y vertical del estrato arbóreo del área de estudio 
Cuadro 2. Especies con mayor índice de valor forestal (ivf) en las cuatro unidades de muestreo en el área de estudio.

\begin{tabular}{|c|c|c|c|c|c|c|}
\hline $\begin{array}{l}\text { UNIDAD } \\
\text { MUESTRAL }\end{array}$ & ESPECIE & $\begin{array}{l}\text { DIÁMETRO } \\
\text { RELATIVO }\end{array}$ & $\begin{array}{c}\text { ALTURA } \\
\text { (RELATIVA) }\end{array}$ & $\begin{array}{l}\text { COBERTURA } \\
\text { (RELATIVA) }\end{array}$ & IVF & $\begin{array}{l}\text { IVF } \\
(\mathrm{ACU})\end{array}$ \\
\hline \multicolumn{7}{|l|}{ UM1 } \\
\hline 1 & $\begin{array}{l}\text { Chrysobalanus icaco } \\
\text { var. pellocarpus }\end{array}$ & 23.15 & 19.78 & 43.48 & 86.42 & 86.42 \\
\hline 2 & Conocarpus erectus & 34.20 & 32.55 & 18.31 & 85.07 & 171.49 \\
\hline 3 & Bucida buceras & 15.32 & 08.44 & 14.69 & 38.47 & 209.96 \\
\hline 4 & Metopium brownei & 13.71 & 12.51 & 4.88 & 31.10 & 241.07 \\
\hline \multirow[t]{2}{*}{5} & Myrica cerifera & 1.58 & 16.42 & 10.35 & 28.36 & 269.44 \\
\hline & Total & 100 & 100 & 100 & 300 & \\
\hline \multicolumn{7}{|l|}{ UM2 } \\
\hline 1 & Bucida buceras & 25.90 & 14.90 & 21.68 & 62.49 & 62.49 \\
\hline 2 & Conocarpus erectus & 21.67 & 23.71 & 15.47 & 60.86 & 123.36 \\
\hline 3 & $\begin{array}{l}\text { Chrysobalanus icaco } \\
\text { var. pellocarpus }\end{array}$ & 14.24 & 15.15 & 18.90 & 48.30 & 171.66 \\
\hline 4 & Manilkara zapota & 11.57 & 12.54 & 09.05 & 33.18 & 204.84 \\
\hline \multirow[t]{2}{*}{5} & Coccoloba cozumelensis & 02.20 & 02.91 & 18.79 & 23.90 & 228.75 \\
\hline & Total & 100 & 100 & 100 & 300 & \\
\hline \multicolumn{7}{|l|}{ UM3 } \\
\hline 1 & Bucida buceras & 37.32 & 27.48 & 41.54 & 106.35 & 106.35 \\
\hline 2 & Conocarpus erectus & 29.92 & 33.01 & 20.46 & 83.39 & 189.74 \\
\hline 3 & Rhizophora mangle & 12.44 & 16.66 & 16.31 & 45.42 & 235.16 \\
\hline 4 & $\begin{array}{l}\text { Chrysobalanus icaco } \\
\text { var. pellocarpus }\end{array}$ & 12.01 & 12.23 & 14.67 & 38.92 & 274.09 \\
\hline \multirow[t]{2}{*}{5} & Myrica cerifera & 02.65 & 03.51 & 02.50 & 08.67 & 282.76 \\
\hline & Total & 100 & 100 & 100 & 300 & \\
\hline \multicolumn{7}{|l|}{ UM4 } \\
\hline 1 & Bucida buceras & 67.97 & 55.45 & 36.77 & 160.20 & 160.20 \\
\hline 2 & $\begin{array}{l}\text { Chrysobalanus icaco } \\
\text { var. pellocarpus }\end{array}$ & 15.75 & 19.13 & 55.68 & 90.57 & 250.78 \\
\hline 3 & Rhizophora mangle & 04.42 & 08.61 & 02.66 & 15.70 & 266.48 \\
\hline 4 & Annona glabra & 04.11 & 06.25 & 01.57 & 11.94 & 278.42 \\
\hline \multirow[t]{2}{*}{5} & Conocarpus erectus & 04.32 & 04.98 & 01.77 & 11.08 & 289.50 \\
\hline & Total & 100 & 100 & 100 & 300 & \\
\hline
\end{tabular}

Acu: Acumulativo.

Fuente: Elaboración propia. 


\section{Diversidad de especies}

Considerado el índice de diversidad de Shannon-Wiener ( $\left.\mathrm{H}^{\prime}\right)$, el valor general de la diversidad encontrada en el área de estudio fue de 2.7; en tanto, por um, la diversidad resultó mayor en la 2 debido principalmente a que el número de individuos de las especies presentes se observó homogéneo, ya que no constituyó la um con mayor cantidad de especies, mientras que la 4 fue la de menor diversidad sobre todo por las pocas especies existentes y la dominancia que manifiestan algunas de ellas (cuadro 3).

Cuadro 3. Valores de riqueza, abundancia y diversidad por unidad de muestreo.

\begin{tabular}{|ccccc|}
\hline UNIDAD DE MUESTREO & RIQUEZA & ABUNDANCIA & DIVERSIDAD $\left(\mathrm{H}^{\prime}\right)$ & EQUIDAD \\
\hline UM1 & 13 & 422 & 2.70 & 0.81 \\
\hline UM2 & 12 & 309 & 3.28 & 0.88 \\
\hline UM3 & 15 & 352 & 2.58 & 0.78 \\
\hline UM4 & 9 & 183 & 2.24 & 0.70 \\
\hline
\end{tabular}

Fuente: Elaboración propia.

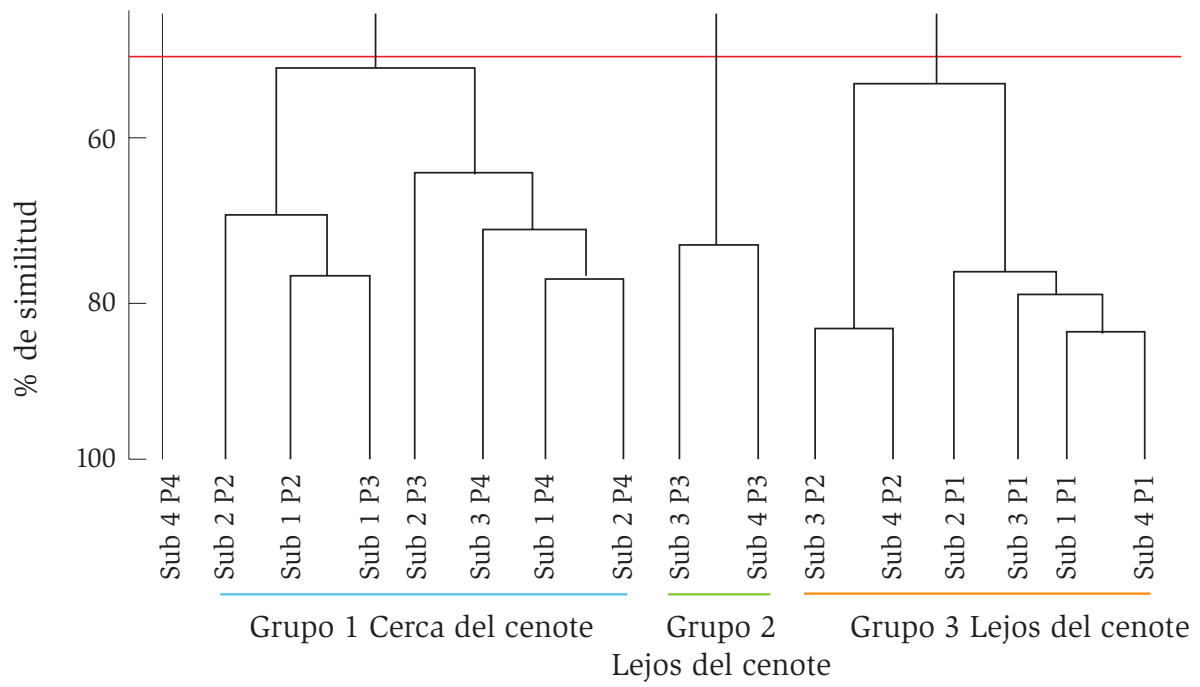

Fuente: Elaboración propia.

Figura 3. Agrupamiento de las subparcelas de las unidades de muestreo en función del índice de similitud Bray-Curtis 
Similitud entre las unidades de muestreo.

En el análisis de agrupamiento se utilizó el índice de Bray-Curtis y se consideraron en cada parcela cuatro subparcelas, separando tres grupos y una subparcela aislada. El primer grupo está conformado por las subparcelas de las um 2, 3 y 4 que se hallan cercanas al cenote; en el segundo se encuentran las subparcelas pertenecientes únicamente a la um3 localizadas lejos del cenote, y en el tercero se incluyen las subparcelas de las um 1 y 2 ubicadas también lejos del cenote (figura 3).

De acuerdo con el SIMPER, considerando un porcentaje acumulativo menor de 90 \% de las especies distintivas por grupos se encontró que Bucida buceras, Chrysobalanus icaco var. pellocarpus, Conocarpus erectus y Rhizophora mangle fueron las especies con mayor presencia en casi todos ellos. Cabe mencionar que el grupo 3 fue el que manifestó el número más bajo de especies distintivas teniendo en cuenta un porcentaje acumulativo menor de 90 \% (cuadro 4). En el

Cuadro 4. Especies distintivas de cada grupo en concordancia con el porcentaje del análisis de similitud (SIMPER), considerando el porcentaje acumulativo menor de $90 \%$

\begin{tabular}{|c|c|c|c|c|c|}
\hline GRUPOS & ESPECIES & PCPUE & PSIM & PCO & PCA \\
\hline \multirow{3}{*}{ Grupo 1} & Bucida buceras & 21.71 & 25.88 & 44.04 & 44.04 \\
\hline & $\begin{array}{l}\text { Chrysobalanus icaco var. } \\
\text { pellocarpus }\end{array}$ & 16.00 & 19.53 & 33.23 & 77.27 \\
\hline & Rhizophora mangle & 04.29 & 05.58 & 09.50 & 86.76 \\
\hline \multirow{4}{*}{ Grupo 2} & Conocarpus erectus & 44.50 & 38.81 & 52.70 & 52.70 \\
\hline & Rhizophora mangle & 28.50 & 22.89 & 31.08 & 83.78 \\
\hline & Conocarpus erectus & 27.83 & 20.38 & 33.46 & 33.46 \\
\hline & Myrica cerifera & 15.00 & 10.48 & 17.20 & 50.66 \\
\hline \multirow{3}{*}{ Grupo 3} & $\begin{array}{l}\text { Chrysobalanus icaco var. } \\
\text { pellocarpus }\end{array}$ & 16.67 & 09.99 & 16.40 & 67.06 \\
\hline & Metopium brownie & 11.83 & 06.42 & 10.54 & 77.60 \\
\hline & Bucida buceras & 09.00 & 05.67 & 09.31 & 86.90 \\
\hline
\end{tabular}

PCPUE = promedio de la abundancia; $\mathrm{PSIM}=$ similitud promedio; $\mathrm{PCO}=$ porcentaje de contribución; PCA = porcentaje de contribución acumulado.

Fuente: Elaboración propia. 
grupo 1, conformado por las subparcelas localizadas cerca del cenote, las especies con mayor contribución en este porcentaje fueron Bucida buceras y Chrysobalanus icaco var. pellocarpus, mientras que en los grupos 2 y 3 , formados por subparcelas ubicadas lejos del cenote, las especies más distintivas para el primero fueron Conocarpus erectus y Rhizophora mangle, y para el segundo, Conocarpus erectus, Myrica cerifera y Chrysobalanus icaco var. pellocarpus (cuadro 4).

\section{Discusión}

En este estudio se registraron 1266 individuos pertenecientes a 16 especies. La mayoría de los individuos exhibió diámetros $<10 \mathrm{~cm}$, mostrándose una distribución agregada entre los individuos debido a que las especies halladas de acuerdo con su ecología determinaban la presencia unas de otras; mientras que para la estructura vertical se obtuvieron tres estratos bien diferenciados, los cuales concuerdan con los reportados por Díaz-Gallegos, Castillo-Acosta y García Gil (2002) en la Reserva de la Biosfera Calakmul, en Campeche, quienes consignaron 65 especies, con alturas de hasta $18 \mathrm{~m}$, en las cuales se presentaron también tres estratos bien definidos. Si bien hay cierta similitud entre algunas especies encontradas en Calakmul con el presente estudio, como $C$. cozumelensis, $M$. brownei, $M$. zapota, entre otras, probablemente se deba a que este estudio se llevó a cabo en zonas bajas inundables. Existe una diferencia significativa entre el número de especies y la altura de los individuos; en esta investigación, la altura máxima fue de $8 \mathrm{~m}$ y la mínima de $2 \mathrm{~m}$, debido principalmente a que en la zona no hay árboles que superen los $10 \mathrm{~m}$. Por último, cabe señalar que, comparando este trabajo con aquel realizado en un rancho en Cozumel por Chan-Quijano (2012), no se observó alguna similitud en el tipo de vegetación predominante en ambos, dadas las características distintas de las zonas de estudio.

Al contrastar este trabajo con el efectuado por Zarco-Espinosa et al. (2010) en la selva mediana perennifolia en Macuspana, Tabasco, se advirtió una diferencia significativa, pues los investigadores registraron 3789 individuos y dos estratos de vegetación (el superior y el inferior). Asimismo, no hubo semejanza en la composición florísticas y los individuos mostraron una distribución aleatoria (índice de Morisita) y presentaron alturas hasta de $27 \mathrm{~m}$. 
En cuanto al Ivf se encontró que las especies con altos valores fueron $B$. buceras, $C$. erectus y $C$. icaco var. pellocarpus; estas son especies que, de acuerdo con su ecología, están presentes en zonas inundables, como el caso del área de estudio. Lo anterior se sustenta en lo hallado por Tun-Dzul et al. (2008) en su estudio de vegetación en selva baja inundable en la Península de Yucatán, puesto que las especies que exhibieron altos índices de valor forestal fueron $B$. buceras, $M$. brownei y $M$. zapota. Además es importante mencionar que las zonas de estudio de estos trabajos mostraron condiciones similares, como los efectos de borde (carretera), la inundación (bajos inundables) y partes de urbanización (construcciones). Por otro lado, en una investigación realizada por Basáñez, Alanís y Badillo (2008) en Papantla, Veracruz, las especies con altos valores forestales fueron Brosimum alicastrum (Swartz), Bursera simaruba L. (Sarg) y Aphananthe monoica (Hemsley), las cuales divergen de las reportadas en este estudio, debido a las diferencias en el tipo de vegetación y a los suelos someros existentes en el sitio de muestreo de Basáñez, Alanís y Badillo, ya que la inundación no perdura como sucede en las zonas bajas inundables.

Por otra parte, aunque en este trabajo se obtuvo la menor riqueza específica y número de individuos dado que únicamente se tomaron individuos $\geq 5 \mathrm{~cm}$ de DAP -en comparación con el de Zarco-Espinosa et al. (2010), donde se midieron individuos $\geq 1 \mathrm{~cm}$ de DAP-, aquí se manifestó el mayor valor de diversidad con 2.7 bits/individuos, el cual fue influido principalmente por la menor dominancia de sus especies presentes. De tal modo se observa que el método de muestreo que afecta a la riqueza de especie y la homogeneidad de sus abundancias repercuten en los valores de diversidad en los estudios de la vegetación. Por ejemplo, en un trabajo llevado a cabo por Vázquez-Negrín et al. (2011) en Tenosique, Tabasco, se encontró una diversidad de 3.6 bits/individuos con un DAP $\geq 2.5 \mathrm{~cm}$, mientras que Godínez-Ibarra y López-Mata (2002), en Santa Gertrudis, Veracruz, advirtieron una diversidad de 3.1 al censar individuos $\geq 1 \mathrm{~cm}$ de DAP.

En el agrupamiento considerando el promedio de similitud se resume que hubo semejanza en subparcelas cercanas al cenote, o sea, las um 2, 3 y 4 compartiendo especies entre ellas, y las um 1 y 2 de acuerdo con las subparcelas lejanas al cenote; esto contrasta con lo reportado por Zarco-Espinosa et al. (2010), quienes emplearon el mismo diseño de muestreo, con la diferencia de que registraron individuos con un DAP $\geq 1 \mathrm{~cm}$ y el lugar donde se realizó el 
estudio era selva mediana perennifolia, observando semejanza entre las um 1 y 2, mientras que la 4 presentó valores muy bajos con respecto a las otras um. En tanto, Vázquez-Negrín et al. (2011) emplearon la misma metodología, pero censando todos los árboles con DAP $\geq 2.5 \mathrm{~cm}$ encontraron que la mayor similitud fue entre las uм 3 у 4, ya que comparten más especies, seguidas por las uм 2 y 4; las diferencias entre los trabajos se deben a que la vegetación es distinta: en el primero se trata de selva baja subperennifolia, y en cuanto al estudio de Vázquez-Negrín et al. (2011), de selva alta perennifolia. Asimismo, las condiciones presentes en cada trabajo son diferentes, el área de estudio tiene factores específicos en cada um (sabana, construcción, carretera y rancho), lo cual indica que lo encontrado depende de la situación en que esté la selva; por ello, las subparcelas cercanas al cenote exhiben similitud entre ellas y las subparcelas lejanas por igual comparten especies características de zonas perturbadas.

\section{Conclusiones}

La distribución de la estructura horizontal de las um fue agregada, mientras que la estructura vertical mostró tres estratos bien definidos: inferior, medio y superior. Las especies con altos índices de valor forestal presentes en las todas las uM fueron B. buceras, $C$. icaco var. pellocarpus y Conocarpus erectus. La um con mayor IVF fue la 4 con las especies B. buceras y $C$. icaco var. pellocarpus a su alrededor. Se observó que el método de muestreo que afecta a la riqueza de especie y la homogeneidad de sus abundancias repercuten en los valores de diversidad en los estudios de la vegetación. Se encontró diferencia entre la diversidad en las um: la 2 manifestó mayor diversidad (3.28 bits/individuo), seguida de la 1 (2.71 bits/individuo) y la 3 (2.58 bits/individuo), debido principalmente a la equidad de las abundancias de las especies. El arreglo espacial de la vegetación mostró que hubo semejanza en las subparcelas cercanas al cenote, esto es, las um 2, 3 y 4 compartieron especies entre ellas, y las um 1 y 2 de acuerdo con las subparcelas lejanas al cenote. Lo anterior fue influido por las características del lugar y los factores específicos en cada um, como la presencia de sabana, construcción, carretera y rancho. 


\section{Fuentes consultadas}

Andrewartha, H. G. y L. C. Birch (1954). The Distribution and Abundance of Animals. Chicago: University of Chicago Press, $782 \mathrm{pp}$.

Arriaga-Cabrera, L., E. Vázquez-Domínguez, J. González-Cano, R. Jiménez-Rosenberg, E. Muñoz-López y V. Aguilar-Sierra (coords.) (1998). Regiones prioritarias marinas de México. México: Comisión Nacional para el Conocimiento y Uso de la Biodiversidad, 198 pp.

Basáñez, A. J., J. L. Alanís y E. Badillo (2008). “Composición florística y estructura arbórea de la selva mediana subperennifolia del ejido 'El Remolino', Papantla, Veracruz”. Avances en Investigación Agropecuaria, 12 (2), 3-21.

Cabrera, E. (1997). "La vegetación en la zona de Xcalak”. Amigos de Sian Ka'an. Boletín, 17, 3-47.

Campos-Cámara, B. L. (1996). "El turismo alternativo como estrategia de integración entre Xcalak, Quintana Roo y San Pedro, Belice. Antecedentes y perspectivas". Tesis de maestría en Ciencias. Chetumal: El Colegio de la Frontera Sur.

Chan-Quijano, J. G. (2012). "Diversidad de siete especies arbóreas en el Rancho la Estrella en la isla de Cozumel, Quintana Roo, México”, en J. G. Chan-Quijano, R. Martínez-Yañez y A. Espinosa-Tenorio (eds.). Manejo sostenible de los recursos naturales: conservación y experiencias. Villahermosa: El Colegio de la Frontera Sur, 86-96.

Clarke, K. R. y R. N. Gorley (2006). PRIMER v6: User Manual/Tutorial. Plymouth: PRIMER-E, $190 \mathrm{pp}$.

Clarke, K. R. y R. M. Warwick (2001). Change in Marine Comunities: An Approach to Statistical Analysis and Interpretation. 2a ed. Plymouth: PRIMER-E, 203 pp.

Cortés-Castelán, J. C. y G. A. Islebe (2005). “Influencia de factores ambientales en la distribución de especies arbóreas en las selvas del sureste de México”. Revista de Biología Tropical, 53 (1-2), 115-133.

Cox, W.G (1981) Laboratory manual of general ecology. Iowa, USA: William C. Brown.

Curtis, J. T. y R. P. McIntosh (1951). "An upland forest continuum in the pariréforest border region of Wisconsin”. Ecology, 32, 476-496.

Dallmeier, F. (ed.). (1992). Long-Term Monitoring of Biological Diversity in Tropical Forest Areas: Methods for Establishment and Inventory of Permanent 
Plots. París: United Nations Educational, Scientific and Cultural Organization (MAB Digest, 11), 72 pp.

Díaz-Gallegos, J., O. Castillo-Acosta y G. García-Gil (2002). “Distribución espacial y estructura arbórea de la selva baja subperennifolia en un ejido de la Reserva de la Biosfera Calakmul, Campeche, México”. Universidad y Ciencia, 18 (35), 11-28.

Godínez-Ibarra, O. y L. López-Mata (2002). “Estructura, composición, riqueza y diversidad de árboles en tres muestras de selva mediana subperennifolia”. Anales del Instituto de Biología, Universidad Nacional Autónoma de México, Serie Botánica, 73 (2), 283-314.

Hubbell, S. P. (1979). "Tree dispersion, abundance, and diversity in a tropical dry forest”. Science, New Series, 203 (4387), 1299-1309.

Hubbell, S. P. y R. B. Foster (1983). "Diversity of canopy trees in a neotropical forest and implications for conservation”, en S. L. Sutton, T. C. Whitmore y A. C. Chadwick (eds.). Tropical Rain Forest: Ecology and Management. Londres: Blackwell Scientific Publications, 25-41.

Jiménez-Bueno, D. (1994). “Distribución de las especies arbóreas en tres comunidades de mangle en la Isla del Carmen, Campeche”. Tesis de licenciatura. Toluca: Universidad Autónoma del Estado de México, 67 pp.

Karousakis, K. (2007). Incentives to Reduce GHG Emissions from Deforestation Lessons Learned from Costa Rica and Mexico. París: Organization for Economic Co-operation and Development, $50 \mathrm{pp}$.

Krebs, C. J. (1978). Ecology. The Experimental Analysis of Distribution and Abundance. Nueva York: Harper \& Row, 678 pp.

Lot, A. y Chiang, F. (1986). Manual de herbario. Administración y manejo de colecciones, técnicas de recolección y preparación de ejemplares botánicos. México: Consejo Nacional de Ciencia y Tecnología.

Medina-González, R. M. (2000). Aspectos biológicos de los cenotes de Yucatán. Mérida: Ediciones de la Universidad de Yucatán/Secretaría de Educación Pública (serie Fauna de los Cenotes de Yucatán, 18), 6 pp.

Moreno-Casasola, P. (1986). "Sand movement as a factor in the distribution of plant communities in a costal dune system". Vegetatio, 65 (2), 67-76.

Mueller-Dombois, D. y Ellenberg, H. (1974). Aims and methods of vegetation ecology, John Wiley and Sons.

Tun-Dzul, F., H. Vester, R. Durán García y B. Schmook (2008). “Estructura ar- 
bórea y variabilidad temporal del NDvi en los 'bajos inundables' de la Península de Yucatán, México”. Polibotánica, 25, 69-90.

Vázquez-Negrín, I., O. Castillo-Acosta, J. I. Valdez-Hernández, J. Zavala-Cruz y J. L. Martínez-Sánchez (2011). "Estructura y composición florística de la selva alta perennifolia en el ejido Niños Héroes Tenosique, Tabasco, México”. Polibotánica, 32, 41-61.

Zaldívar-Jiménez, M. A. (1999). "Estructura de la comunidad de manglar y su relación con las características físicas y químicas del sedimento en la Ciénaga Sisal-Chuburná, Yucatán, México”. Tesis profesional. Mérida: Universidad Autónoma de Yucatán/Instituto Politécnico Nacional-Centro de Investigación y de Estudios Avanzados, Unidad Mérida-Laboratorio de Producción Primaria, 102 pp.

Zarco-Espinosa, V. M., J. I. Valdez-Hernández, G. Ángeles-Pérez y O. CastilloAcosta (2010). "Estructura y diversidad de la vegetación arbórea del Parque Estatal Agua Blanca, Macuspana, Tabasco”. Universidad y Ciencia, 26 (1), 1-17. 
